\title{
Neoliberalismo, desempeño económico y mercados laborales en Latinoamérica: un enfoque comparativo*
}

Neoliberalism, economic performance and labor markets in Latin America: a comparative approach

\section{Neoliberalismo, desempenho econômico e mercados laborais em Latino América: um enfoque comparativo}

Roberto Zepeda Martínez** - México

Recibido el 5 de diciembre de 2012, aceptado el 16 de septiembre de 2013

* Este artículo corresponde a los resultados de la investigación terminada sobre neoliberalismo y mercados laborales en Latinoamérica, realizada en la Universidad Nacional Autónoma de México, la Universidad de Sheffield Inglaterra y la Universidad del Mar, Campus Huatulco (2IEI1302), en el periodo 2009-2013.

** Licenciado en Ciencias de la Comunicación. Doctor en Ciencias Políticas y Sociales. ProfesorInvestigador de la Universidad del Mar, Campus Huatulco (México). Correo electrónico: zepeda_ roberto@yahoo.com 


\title{
Resumen
}

Objetivos: identificar las premisas centrales del neoliberalismo y sus repercusiones en las esferas económica y laboral. Además, detectar las fluctuaciones del empleo informal, desempleo y sindicalización en Latinoamérica desde la década de los 80 s, con el propósito de realizar un análisis crítico y evaluar los resultados de las políticas neoliberales aplicadas en la región. Metodología: se hizo una investigación documental sobre neoliberalismo y sus principales fundamentos. Se complementó con una investigación cuantitativa enfocada en el análisis longitudinal de datos estadísticos generados por organismos internacionales para detectar los niveles de empleo informal, desempleo y sindicalización en Latinoamérica. Se seleccionó un grupo de 11 países en la región latinoamericana para detectar convergencias y divergencias. Resultados: se evidencia una divergencia notable en el desempeño económico y las características de los mercados laborales entre los países examinados. Sin embargo, es evidente la desaceleración económica desde los 80 . México se ubica en los últimos lugares en crecimiento económico en la primera década de 2000. Brasil y Chile han presentado un notable crecimiento económico en el periodo neoliberal, aunque la desigualdad social aún es significativa. El desempleo urbano más alto se encuentra en Colombia y en países con Seguro de Desempleo, como Argentina, Chile y Uruguay. Argentina es el país más sindicalizado en la región. Conclusiones: a pesar de que la mayoría de los países en Latinoamérica ha implantado un modelo económico neoliberal desde finales de los setenta, aún persisten rezagos en el desempeño económico y la creación de empleos bien remunerados. No obstante, las divergencias encontradas no permiten establecer una relación directa entre el neoliberalismo, el desempeño económico y los mercados laborales.

Palabras claves: Neoliberalismo, Latinoamérica, Mercados laborales, Sindicalismo.

\begin{abstract}
Objectives: To identify the central premises of neoliberalism and its impact on economic and employment areas. To detect fluctuations in informal employment, unemployment and unionization in Latin America since the 80's in order to critically analyze and evaluate the results of neoliberal policies in the region. Methodology: Documentary research was carried out on neoliberalism and its main foundations, which was complemented by quantitative research focused on the longitudinal analysis of statistical data generated by international agencies to detect the levels of informal employment, unemployment and unionization in Latin America. We selected a group of 11 countries in Latin America to identify convergences and divergences. Results: There is a significant divergence in economic performance and characteristics of labor

14 markets amongst the countries examined as evidence. However, economic slowdown
\end{abstract}


is evident since 1980. Mexico ranks near the bottom in economic growth in the 2000's. Brazil and Chile have shown a remarkable economic growth in the neoliberal period, although social inequality is still significant. The highest urban unemployment is in Colombia and in countries with unemployment insurance, such as Argentina, Chile and Uruguay. Argentina is the most unionized country in the region. Conclusions: Although most countries in Latin America have implemented a neoliberal economic model since the late 70's, it still lags behind in economic performance and in the creation of well-paying jobs. However, the differences that were found did not allow us to establish a direct relationship between neoliberalism, economic performance and labor markets.

Keywords: Neoliberalism, Latin American Labor Markets, Trade Unionism.

\section{Resumo}

Objetivos: identificar as premissas centrais do neoliberalismo e suas repercutes nas esferas econômica e laboral. Além, detectar as flutuações do emprego informal, desemprego e sindicalização em Latino América desde a década dos 80s, com o propósito de realizar uma análise crítico e avaliar os resultados das políticas neoliberais aplicadas na região. Metodologia: se fez uma pesquisa documental sobre neoliberalismo e seus principais fundamentos. Complementou-se com uma pesquisa quantitativa enfocada na análise longitudinal de dados estatísticos gerados por organismos internacionais para detectar os níveis de emprego informal, desemprego e sindicalização em Latino América. Selecionou se um grupo de 11 países na região latino americana para detectar convergências e divergências. Resultados: se evidencia uma divergência consideravél no desempenho econômico e as características dos mercados laborais entre os países examinados. Contudo, é evidente a desaceleração econômica desde os 80. México localizam se os últimos lugares em crescimento econômico na primeira década de 2000 . Brasil e Chile têm apresentado um considerável crescimento econômico no período neoliberal, enquanto a desigualdade social, contudo é significativa. O desemprego urbano mais alto se encontra em Colômbia e em países com Seguro de Desemprego, como Argentina, Chile e Uruguai. Argentina é o país mais sindicalizado na região. Conclusões: a pesar de que a maioria dos países em Latino América tem implantado um modelo econômico neoliberal desde finais dos $70 \mathrm{~s}$, ainda persistem defasagens no desempenho econômico e a criação de empregos bem remunerados. Não obstante, as divergências encontradas não permitem estabelecer uma relação direta entre o neoliberalismo, o desempenho econômico e os mercados laborais.

Palavras chave: Neoliberalismo, Latino América, Mercados laborais, Sindicalismo.

Para 


\section{Introducción}

Durante el último cuarto del siglo XX, Latinoamérica experimentó una transición económica que se orientó a la aplicación de políticas neoliberales (Cook, 2002; González, 2008). Esto propició diversos resultados en la región, que distan mucho de los postulados originales de esta doctrina económica. El neoliberalismo parte de la premisa de que las fuerzas del mercado traerán como consecuencia una exacta distribución de la riqueza. De contera, la premisa indica que el neoliberalismo es el mejor método para lograr el desarrollo económico y social y que, a la postre, reducirá los índices de desigualdad y pobreza, en virtud de que el libre comercio hará más competitivos a los países, aumentará la productividad y, en este sentido, se crearán más empleos y mejor remunerados (Heywood, 2007; Williamson, 2000).

Pero, si se tienen en cuenta los resultados que ha causado el neoliberalismo en diversos países en Latinoamérica, se constata un panorama desolador: bajo crecimiento económico, aumento de la brecha entre ricos y pobres, desempleo constante, aumento de la informalidad, desmantelamiento del Estado Benefactor, así como el declive del sindicalismo y la desarticulación de los movimientos obreros (Harvey, 2005; Saad-Filho, 2005).

Diversos trabajos académicos han abordado las consecuencias de este proceso en la región. Sin embargo, el presente artículo está limitado a las repercusiones en el desempeño económico (medido por las fluctuaciones del Producto Interno Bruto -PIB) y las características y evolución de la fuerza laboral, con el propósito de determinar los efectos de las políticas neoliberales en Latinoamérica. En este artículo se advierte la transformación del modelo económico hacia un modelo neoliberal en la mayoría de los países latinoamericanos. Posteriormente, se analizan una serie de datos que dan cuenta del desempeño económico y la esfera laboral. Finalmente, se consideran las evidencias encontradas y en las conclusiones se dilucidan las repercusiones del neoliberalismo en tales esferas.

\section{Metodología}

Esta investigación se basó en una combinación de los métodos cuantitativo y cualitativo. Esta estrategia fue crucial para obtener diferentes tipos de información. Por una parte, la investigación cualitativa comprendió el análisis documental con el objetivo de examinar conceptos clave que fueron vinculados entre sí. 
La estrategia consistió en desfragmentar el concepto del neoliberalismo en sus componentes más relevantes para determinar sus efectos de manera más exacta. Las categorías de análisis fueron: políticas neoliberales (flexibilización, privatización y globalización), así como las características del neoliberalismo y su proceso de implantación en Latinoamérica. En tal propósito, la estrategia metodológica se enfocó en el análisis de fuentes primarias y secundarias (libros, artículos, y publicaciones de organismos nacionales e internacionales) que se abordaron considerando las categorías señaladas para propiciar una mejor comprensión en dos aspectos: la relación del neoliberalismo con el desempeño económico y con los mercados laborales.

Por otra parte, la investigación fue complementada con un análisis cuantitativo, considerando información estadística para explorar la relación entre variables, algunas de las cuales usualmente se miden cuantitativamente (Hague y Harrop, 2001, p. 89). Las variables analizadas fueron: desempeño económico (fluctuaciones del PIB), niveles de desigualdad (coeficiente de Gini), tasa de desempleo total y urbano, empleo informal dentro de la fuerza laboral y tasa de sindicalización.

Con el objetivo de analizar la evolución de tales indicadores y de las esferas económica y laboral, se accedió a bases de datos primarios de organismos internacionales como la Comisión Económica para América Latina y el Caribe (CEPAL/ECLAC), la Organización Internacional del Trabajo (OIT/ILO), la Conferencia de las Naciones Unidas sobre Comercio y Desarrollo (CNUCyD/ UNCTAD). Cuando las fuentes primarias no facilitaron los datos necesarios, se hizo uso de fuentes secundarias que suministraran información necesaria en las áreas señaladas. Los datos reunidos fueron útiles para generar patrones, fluctuaciones y comparaciones para dilucidar el desempeño económico y las características de los mercados laborales.

En el análisis de tales indicadores, se aplicó un análisis cuantitativo longitudinal para evaluar la evolución de estos indicadores. Asimismo, se profundizó en la interrelación entre las políticas económicas neoliberales, el desempeño económico y los mercados laborales. En cuanto a los países del estudio, se considera un grupo de 11 países que fueron seleccionados de acuerdo con su relevancia económica en la región y su pertenencia a bloques económicos (Mercosur ${ }^{1}$,

1 Mercado Común del Sur. Países integrantes: Brasil, Argentina, Uruguay, Venezuela y Paraguay (Venezuela ingreso en Julio de 2012, después de Paraguay fue excluido temporalmente por la cláusula democrática). Año de inicio: 1993. 
Comunidad Andina ${ }^{2}$ y TLCAN³). Estos países son: Brasil, México, Argentina, Colombia, Perú, Venezuela, Ecuador, Chile 4 , Bolivia, Paraguay y Uruguay. Se excluyen países del Caribe y Centroamérica.

\section{Resultados}

Las políticas neoliberales coincidieron en general con las bajas tasas de crecimiento económico, la expansión del empleo informal y el desempleo, así como la caída en las tasas de sindicalización en la región. En este escenario, las reformas neoliberales no se tradujeron en una mejoría para la mayoría de los trabajadores que vieron reducirse sus niveles de bienestar. Obviamente, hay un paisaje diverso en la región, pero en términos generales el panorama sugiere que en la mayoría de los casos no se ha aplicado una política económica que genere crecimiento y mejores empleos, y propicie un entorno favorable a la clase trabajadora.

El neoliberalismo se tradujo en políticas concretas como la privatización, la flexibilización de los mercados laborales y la globalización manifestada por medio de la integración económica y el libre comercio. Los países de la región adoptaron progresivamente estas prescripciones que se convirtieron en la base de sus políticas económicas, pero que mostraron resultados poco favorables en términos de crecimiento económico y de creación de empleos, y contribuyeron al aumento de la desigualdad en región. Tales aseveraciones se demuestran y se discuten en los siguientes apartados.

\section{Neoliberalismo}

El neoliberalismo ha ganado un avance significativo en décadas recientes como la doctrina económica preponderante en la mayoría de los países capitalistas. Una de las definiciones más apropiadas es descrita por David Harvey (2005):

El neo-liberalismo es, en primer término, una teoría de prácticas de política económica que propone que el bienestar de la humanidad se puede lograr de mejor forma, liberando las libertades emprendedoras y las habilidades del individuo dentro de una estructura institucional caracterizada por fuertes

2 Países integrantes: Colombia, Ecuador, Perú y Bolivia. Año de inicio: 1969.

3 Tratado de Libre Comercio de América del Norte. Países integrantes: México, Estados Unidos y Canadá. Año de inicio: 1994.

4 Chile tiene tratados de libre comercio con 50 países, por ejemplo, con Canadá, México, Centroamérica, Unión Europea, Estados Unidos, Corea y China, entre otros.

Para $\mid$ Zepeda Martínez, Roberto (2013). Neoliberalismo, desempeño económico y mercados 
derechos de propiedad privada, libre mercado, y libre comercio. El rol del estado es crear y preservar una estructura institucional apropiada para tales prácticas. Aún más, si los mercados no existen (en áreas como la tierra, el agua, educación, cuidado de la salud, seguridad social, o contaminación ambiental), entonces, se deben de crear, con la acción del Estado, si es necesario. Pero más allá de estas tareas, el Estado no debería de involucrarse. La intervención del Estado en los mercados (una vez creados) debe ser mantenida al mínimo (p. 3) (traducción propia).

Las afirmaciones de Harvey resultan esenciales para evaluar y comprender la dimensión exacta del neoliberalismo, así como los efectos que ha sufrido la clase trabajadora en varios países, incluyendo la región latinoamericana. En este sentido, la "restauración del poder de clase" generada por el neoliberalismo, destacada por Harvey como una de las principales fuerzas detrás del proceso, implica la existencia de élites económicas nacionales que contribuyeron a la introducción de las reformas neoliberales y que resultaron favorecidas, por ejemplo, en el proceso de privatización, uno de los preceptos de esta doctrina.

En este marco de ideas, se considera al neoliberalismo, no sólo como una serie de prescripciones de política económica para el desarrollo económico, sino como una doctrina ideológica impuesta por las clases dominantes y las élites económicas con el propósito de recuperar poder e influencia.

El neoliberalismo ha generado efectos negativos en los mercados laborales, debido, principalmente, a tres factores. Primero, las políticas neoliberales han estimulado el proceso de globalización económica, favoreciendo la expansión del capitalismo más allá de las fronteras nacionales y promoviendo, como inevitable dogma, una serie de prescripciones en política pública para las naciones emergentes y favoreciendo a las corporaciones multinacionales (Hirst y Thompson, 2003).

Segundo, el neoliberalismo ha hecho a los mercados laborales más flexibles, mediante la desregulación de los contratos colectivos; esta situación ha reducido los salarios reales y suprimido los beneficios laborales que los trabajadores habían conquistado por medio de luchas violentas en décadas pasadas (Gamble, 2001), generando una precarización del trabajo. Tercero, el neoliberalismo ha pugnado por la relativa reducción del gobierno en la regulación de la economía (Heywood, 2007), se ha alentado la privatización de numerosas empresas paraestatales y el Estado ha abandonado la provisión de la seguridad social y de otros beneficios sociales para la clase trabajadora. Como resultado, el empleo formal y permanente se reduce y durante el proceso de privatización de empresas públicas se flexibilizan los contratos colectivos (Saad-Filho, 2005).

Para citar este artículo:
Zepeda Martínez, Roberto (2013). Neoliberalismo, desempeño económico y mercados laborales en Latinoamérica: un enfoque comparativo. Ánfora 20(35), 13-40. Universidad Autónoma de Manizales. ISSN 0121-6538. 
En México, el proceso de privatización fue significativo para el desmantelamiento de contratos colectivos nacionales y el debilitamiento de los sindicatos. Durante el periodo 1982-1994, alrededor del 80\% de las empresas paraestatales fueron privatizadas (MacLeod, 2005, pp. 42-45). En este proceso, la membresía sindical en sectores tales como la minería, el acero, los ferrocarriles, entre otros, se erosionó drásticamente.

Las políticas neoliberales, por otra parte, comprenden la estabilización de los indicadores macroeconómicos, particularmente el control y reducción de la tasa de inflación y el déficit público. Mientras que el control de la inflación fue una de las prioridades de los gobiernos latinoamericanos, otros aspectos, tales como la promoción del empleo, fueron relegados, y de esta manera impactaron en la creación de empleos formales, más notablemente en el sector público. Asimismo, como ha sido resaltado por diversos analistas, en algunos casos como en México, la consecución de este objetivo se logró exitosamente, aunque esto implicó la contención de los salarios, lo cual erosionó el salario promedio real de los trabajadores.

Además, la reducción de los déficits públicos se logró mediante recortes al gasto social, incluyendo los subsidios a productos básicos alimenticios y en otras áreas, lo cual fue especialmente perjudicial para los niveles de vida de la clase trabajadora, en la primera fase de las reformas neoliberales en la década de los ochenta. En el modelo económico Keynesiano o en el modelo de Industrialización por Sustitución de Importaciones (ISI), el Estado se apoyaba en las organizaciones sindicales para controlar la inflación mediante los topes salariales, mientras que en el modelo neoliberal, los recortes en el gasto público resultaron más importantes al respecto.

De esta manera, el neoliberalismo no sólo se convirtió en el paradigma predominante desde los 80, sino que también generó grandes repercusiones en las políticas públicas, tales como la supresión de barreras al comercio internacional, la desregulación de los mercados (incluyendo el mercado laboral) y ha apoyado el retiro de la intervención del Estado en la economía. Los pilares centrales del neoliberalismo son el mercado y lo individual (Heywood, 2007, p. 52). Sin embargo, considerando las experiencias en diferentes países en donde las reformas neoliberales se han aplicado, se podría sugerir que los resultados han sido desalentadores en términos de crecimiento económico y composición del empleo, aunque estos resultados difieren en gran medida, dependiendo del contexto político y económico nacional. 
Aunque las ideas neoliberales habían sido promovidas por un grupo de académicos desde la década del 40 del siglo pasado, fue en la década de los ocheta cuando éstas fueron implementadas y promovidas, principalmente por Ronald Reagan en los Estados Unidos y Margaret Thatcher en el Reino Unido; aunque desde 1976 los "Chicago Boys" las habían aplicado en Chile por encargo de Pinochet. Subsecuentemente, la mayoría de los países desarrollados empezaron a adoptarlas gradualmente. De igual manera, estas políticas fueron impuestas por instituciones supranacionales como el Fondo Monetario Internacional y el Banco Mundial en la mayoría de los países en desarrollo en Latinoamérica, entre ellos México.

Desde la década de los treinta, el modelo de Industrialización por Sustitución de Importaciones (ISI), fue promovido por la mayoría de los países en Latinoamérica. Este modelo estuvo caracterizado por una economía relativamente cerrada a los mercados internacionales, complementada con una fuerte participación del Estado en la esfera económica, como propietario de paraestatales en sectores estratégicos; el sector industrial fue el motor del crecimiento económico y el empleo (Stallings y Peres, 2000, p. 37). La prosperidad económica de estas décadas permitió el fomento al empleo, la seguridad social y altas tasas de sindicalización lo que se traducía en mayores estándares laborales y sociales para los trabajadores. Cuando el modelo de ISI mostró signos de agotamiento en los 70 y 80 , fue gradualmente sustituido por el modelo neoliberal.

A finales de los setenta y principios de los ochenta, Latinoamérica tenía grandes déficits de cuenta corriente, debido al desplome de los precios de las materias primas de exportación (entre ellas el petróleo), y al aumento de las tasas de interés en el ámbito internacional, lo que acrecentó los pagos por la deuda externa.

Latinoamérica, de pronto, se vio sumergida en una aguda crisis social y económica: El PIB per cápita en la región se redujo en 8\%, mientras que los salarios reales cayeron 17\% entre 1982-1983; el gasto social per cápita se desplomó 10\% en términos reales entre 1982 y 1986. Además, en muchos países se presentó una hiperinflación: en Bolivia la tasa de inflación fue de 8,170\% en 1985; en Perú 7,649\% en 1990; 4,924\% en Argentina en 1989 y 2,500\% en Brasil en 1993. La fuga de capitales también fue notable: entre 1979 y 1982, alcanzó \$26.5 billones de dólares en México; $\$ 22$ billones en Venezuela y $\$ 19.2$ billones en Argentina (Kirby, 2003, p. 52). Lo anterior se tradujo en "la década perdida" en términos de crecimiento y desarrollo económico.

Después de la crisis económica desatada en 1982 en México, el presidente Miguel de la Madrid adoptó el neoliberalismo como condición impuesta por los 
organismos financieros internacionales para liberar los préstamos monetarios (Harvey, 2005, p. 29). De hecho, las élites económicas domésticas fortalecieron el proceso de neoliberalización. Gamble (2000) destaca que la adopción del neoliberalismo no se dio sólo como resultado de la imposición a los países en desarrollo, por los organismos internacionales dominadas por países estratégicos, sino que también es el resultado del apoyo de las élites económicas y políticas nacionales. De igual manera, Harvey (2005) coincide con Gamble y destaca a dos países de Latinoamérica, México y Chile, donde las políticas neoliberales fueron impuestas de diferente manera por los organismos internacionales.

Hacia finales de la década de los 80, el neoliberalismo fue reforzado con el "Consenso de Washington", diseñado por el economista inglés John Williamson, que se refiere a los temas de ajuste estructural que formaron parte de los programas del Banco Mundial y del Banco Interamericano de Desarrollo, entre otras instituciones, en la época de ajuste económico después de la crisis de la deuda desatada en 1982. Algunos se refieren a la "Agenda de Washington", otros a la "Convergencia de Washington" y unos pocos la llaman la "Agenda Neoliberal"5.

Pero, ateniéndose a los resultados que ha causado el neoliberalismo en diversos países (principalmente en Latinoamérica), se evidencia un panorama desolador: bajo crecimiento económico, aumento del desempleo, aumento de la informalidad en los empleos, así como el declive del sindicalismo y la desarticulación de los movimientos obreros. El neoliberalismo explota la fuerza de trabajo barata y se aprovecha de los mercados de los países subdesarrollados, eliminando los aparatos productivos nacionales en donde se presenta.

Incluso, un análisis histórico permite ver que los países más desarrollados no llegaron a ser ricos mediante la aplicación rigurosa de las políticas neoliberales; los periodos de rápido crecimiento económico, tanto en países pobres como ricos, no han coincidido con el neoliberalismo; las políticas asociadas con el rápido crecimiento económico en Latinoamérica (1930-82), Asia del este (1960-98) y China (1978 hasta el presente) contradicen rotundamente las prescripciones del Consenso de Washington en varios aspectos (Saad-Filho, 2005, p. 116).

Como ha sido señalado previamente, las políticas neoliberales fueron adoptadas en la región desde los 70, en el caso de Chile, y para la década de los 90

\footnotetext{
5 Estos son los preceptos del Consenso de Washington: (1) Disciplina fiscal, (2) La inflación como parámetro central de la economía, (3) Prioridades en el gasto público, (4) Reforma Tributaria, (5) Tasas de interés, (6) Tipo de cambio, (7) Política comercial, (8) Inversión Extranjera Directa (IED),

(9) Privatizaciones, (10) Desregulación. Para mayores detalles, véase Williamson, 2000.
}

Para
Zepeda Martínez, Roberto (2013). Neoliberalismo, desempeño económico y mercados laborales en Latinoamérica: un enfoque comparativo. Ánfora 20(35), 13-40. Universidad Autónoma de Manizales. ISSN 0121-6538. 
la mayoría de los países había implementado en gran medida las llamadas políticas neoliberales (Cuadro 1). La aplicación de estas políticas también coincide con la desaceleración de la economía y con la expansión del empleo informal. Algunos países modificaron las políticas del Estado en materia social y, por ende, recortaron subsidios a los alimentos y otros rubros, con lo que perjudicaron a la clase trabajadora.

Como se aprecia en el Cuadro 1, el inicio de las reformas neoliberales en la región empezó con Chile en 1976, le siguieron Bolivia (1985), México (1986), Argentina y Venezuela (1989) durante la década de los ochenta. En la siguiente década, hicieron lo propio países como Colombia y Ecuador, ambos en 1990, Paraguay y Uruguay en 1991, Perú en 1992 y Brasil en 1994, considerando únicamente a los países analizados aquí. Es decir, en un periodo de 10 años (19851994) muchos países se adaptaron al modelo neoliberal en un contexto más o menos democrático, y solo Chile se anticipó a esta dinámica en los setenta, bajo un gobierno autoritario de tipo militar, emergido de un golpe de Estado.

Para algunos autores, el neoliberalismo coadyuvó al proceso de democratización en Latinoamérica. Weyland (2004, pp. 137-138) argumenta que el neoliberalismo ha incidido en el mantenimiento de la democracia, pero ha afectado la calidad de la democracia en la mayoría de los países de la región. Sobre lo primero, argumenta que la integración de Latinoamérica a la economía global ha hecho que la región sea más susceptible a las presiones internacionales para mantener la democracia. Este mismo autor señala que las políticas neoliberales se han implementado tanto por países con regímenes autoritarios como por países semidemocráticos y democráticos, por lo que no se advierte una relación estrecha entre la implementación de las reformas neoliberales y los regímenes políticos.

Para $\mid$ Zepeda Martínez, Roberto (2013). Neoliberalismo, desempeño económico y mercados 


\section{Cuadro 1.- Año de inicio de las reformas neoliberales en Latinoamérica}

\begin{tabular}{|l|c|}
\hline \multicolumn{1}{|c|}{ País } & Año \\
\hline Chile & 1976 \\
\hline Bolivia & 1985 \\
\hline México & 1986 \\
\hline Argentina & 1989 \\
\hline Venezuela & 1989 \\
\hline Colombia & 1990 \\
\hline Ecuador & 1990 \\
\hline Paraguay & 1991 \\
\hline Uruguay & $1973 / 1991$ \\
\hline Perú & 1992 \\
\hline Brasil & 1994 \\
\hline
\end{tabular}

Fuente: el autor con información de María Lorena Cook (1998).

\section{Tasas de crecimiento económico en Latinoamérica}

El advenimiento de la globalización neoliberal durante el periodo 1980-2010 trajo consigo diversos efectos en Latinoamérica. Por ejemplo, en términos económicos, la mayoría de los países atestiguó una baja en el crecimiento del PIB, ya que en la mayoría se produjo una crisis económica en los 80 que causó lo que muchos llaman la década perdida. En contraste con las décadas anteriores, la caída fue notable. Concretamente en el periodo 1965-80, el crecimiento porcentual del PIB en Latinoamérica avanzó en promedio 6.1\%, mientras que en los ochentas apenas creció 1.6\% en total (Robinson, 2004, p. 138). Evidentemente, hay divergencias entre cada uno de los países de la región durante el periodo neoliberal; por ejemplo, para Brasil, Chile y Colombia esta década fue de crecimiento por encima del conjunto de países en la región, lo contrario en los casos de Argentina, Perú y Venezuela, que tuvieron un desempeño económico negativo en este período (UNCTAD, 2009, p. 400).

En la década de los 90, Latinoamérica creció en promedio 3.3\% (ECLAC, 24 2000, p. 85). Se puede decir que la mayoría de los países tuvo una década positi- 
va en términos de crecimiento económico. De acuerdo con datos de la CEPAL, El PIB de México creció 3.5\% en esta década, por encima del promedio, así como el de Argentina que tuvo una expansión del 4.2\% en este periodo. Otros países que crecieron por encima de la media regional fueron Chile (6.6\%), Bolivia (3.8\%), y Perú (4.2\%). En contraste, el desempeño de Brasil en esta década fue menor que el promedio general, ya que el PIB se expandió sólo 2.6\% en total. Otros países que tuvieron tasas de crecimiento por debajo del promedio fueron: Uruguay (3.0\%), Colombia (2.6\%), Paraguay (2.2\%), Venezuela (2.0\%) y Ecuador $(1.7 \%)$, entre otros.

Durante la primera década del siglo XXI, las tasas de crecimiento económico en la región en conjunto han continuado prácticamente en los mismos parámetros, comparado con las tendencias presentadas en los noventa. De esta manera, las cifras permiten evidenciar que la región ha crecido en promedio $3.7 \%$ en el periodo 2001-2010, según cálculos propios con base en datos oficiales de la CEPAL. De acuerdo con otra fuente consultada (UNCTAD, 2012, pp. 424425), los países que registraron un crecimiento por encima del promedio en este periodo fueron: Perú (6.1\%), Argentina (5.6\%), Ecuador (4.6\%), Venezuela (4.6\%), Colombia (4.5\%), Chile (4.0\%), Uruguay (3.9\%) y Paraguay (3.8\%). En contraparte, con base en la misma fuente, los países que tuvieron un desempeño similar o por debajo del crecimiento promedio de la región de Latinoamérica en el periodo (2000-2010) fueron: Brasil (3.7\%) y México (2.1\%). Como se puede ver, el país con menor crecimiento económico entre este grupo de países en la primera década de 2000, fue México.

Datos proporcionados por la CEPAL $(2000,2012)$ y la UNCTAD (2012), permiten analizar el crecimiento económico promedio en las últimas tres décadas en Latinoamérica. Estos datos muestran que Chile obtuvo tasas de crecimiento por encima de la media. Brasil tuvo un desempeño muy similar al presentado en la región en los ochenta $(1.6 \%)$ y una tasa modesta, por debajo del promedio, en los noventa (2.6\% contra 3.3\% respectivamente); pero en el 2000 se recuperó con tasas similar al nivel general en la región. Argentina, por su parte, registró una tasa negativa $(-0.7 \%)$ en la década perdida de los 80 , mientras que en los 90 creció por encima del total de la región (4.2\% contra 3.3\%).

Recientemente, en el periodo 2000-2010, el desempeño económico de Argentina ha sobrepasado a la región con una tasa promedio de aumento del 5.6 $\%$, por encima del promedio general en la región, que fue de $3.7 \%$. Por su parte, México creció con tasas ligeramente por encima del promedio en los 80 y 90 ,

Para $\mid$ Zepeda Martínez, Roberto (2013). Neoliberalismo, desempeño económico y mercados 
aunque en la primera década de 2000 su ritmo de crecimiento $(2.1 \%)$ fue menor al de la región. Chile presenta tasas de crecimiento de casi el doble de la media de los demás países. En los 80 y 90 su economía creció constantemente, casi ininterrumpidamente, con tasas promedio de $3.0 \%$ y $6.6 \%$, respectivamente en estas décadas.

El desempeño económico durante el periodo 1980-2010 fue menor al experimentado en el de 1965-1980. Durante el periodo 1980-2010, Latinoamérica creció en promedio $2.4 \%$ (cálculos propios), muy por debajo del $6.1 \%$ del periodo 1965-1980 (Robinson, 2004, p. 139). De acuerdo con datos de la UNCTAD (2012, pp. 413-416) y la ECLAC (2000), dentro del grupo de países examinados, el país que registró una tasa mayor de crecimiento en el periodo 1980-2010 fue Chile con el 4.5\%, le sigue Colombia, con el 3.6\%, después Argentina, Perú y Paraguay, todos con el 3\%; después Bolivia y Ecuador, ambos con 2.7\%. Brasil $(2.6 \%)$ y México $(2.5 \%)$. Finalmente, con tasas de crecimiento por debajo de la región en este periodo se encuentra Uruguay $(2.3 \%)$ y Venezuela (2\%). En el año 2011, el crecimiento promedio en estos países fue de 5.6\% en promedio, un porcentaje alto de crecimiento, lo cual sugiere que Latinoamérica se está beneficiando de la crisis económica que azota Europa (Cuadro 2).

\section{Cuadro 2.- Tasas de crecimiento promedio del PIB en Latinoamérica}

\begin{tabular}{|l|c|c|c|c|c|}
\hline \multicolumn{1}{|c|}{ Periodo } & $1981-1990$ & $1991-2000$ & $2000-2010$ & $1980-2010$ & 2011 \\
\hline Chile & 3 & 6.6 & 4 & 4.5 & 6 \\
\hline Colombia & 3.7 & 2.6 & 4.5 & 3.6 & 5.9 \\
\hline Argentina & -0.7 & 4.2 & 5.6 & 3.0 & 8.9 \\
\hline Perú & -1.2 & 4.2 & 6.1 & 3.0 & 6.9 \\
\hline Paraguay & 3 & 2.2 & 3.8 & 3.0 & 4.4 \\
\hline Bolivia & 0.2 & 3.8 & 4.1 & 2.7 & 5.2 \\
\hline Ecuador & 1.7 & 1.7 & 4.6 & 2.7 & 7.8 \\
\hline Brasil & 1.6 & 2.6 & 3.7 & 2.6 & 2.7 \\
\hline México & 1.9 & 3.5 & 2.1 & 2.5 & 3.9 \\
\hline Uruguay & 0 & 3 & 3.9 & 2.3 & 5.7 \\
\hline Venezuela & -0.7 & 2 & 4.6 & 2.0 & 4.2 \\
\hline
\end{tabular}

Fuente: Datos de 1981-1990 y 1991-2000 obtenidos de ECLAC, 2000 p. 85; 2012. Datos de 2000-2010, y 2011 (UNCTAD, 2012, pp. 413-416).

Para
Zepeda Martínez, Roberto (2013). Neoliberalismo, desempeño económico y mercados laborales en Latinoamérica: un enfoque comparativo. Ánfora 20(35), 13-40. Universidad Autónoma de Manizales. ISSN 0121-6538. 
Los datos sobre crecimiento económico permiten destacar que el ritmo de crecimiento en la región latinoamericana se desaceleró en las últimas dos décadas del siglo XX y la primera década del siglo XXI, comparado con las décadas inmediatamente anteriores, aunque en la segunda década del presente siglo se ven signos positivos.

El periodo de desaceleración económica coincide con la amplia aplicación de políticas económicas conocidas como neoliberales, aun cuando uno de los dogmas centrales de estas políticas era generar un mayor crecimiento económico, lo cual no se ha concretado.

Aun cuando México ha presentado tasas de crecimiento muy similares a las manifestadas en la región en las últimas décadas, podemos subrayar que en años recientes, concretamente en el periodo 2000-2010, la expansión económica ha sido menor que el promedio de los países en el contorno latinoamericano.

No obstante, se puede afirmar categóricamente que la aplicación de políticas neoliberales ha contribuido a generar desaceleración económica en todos los países, ya que como los datos muestran, en el periodo 1980-2010, países como Chile y Brasil han crecido muy por encima del total en la región. Por lo tanto, el contexto nacional en el que se aplicaron estas políticas es determinante en los resultados.

Además, habría que considerar los niveles de desigualdad que prevalecen en la región. Desde la década de los ochenta, la región Latinoamericana ha tenido los mayores niveles de desigualdad en la distribución del ingreso en el mundo. López-Calva y Lustig (2010, pp. 2-3) indican que hacia mediados de 2000, Latinoamérica era 18\% más desigual que África Subsahariana, 36\% más desigual que Asia del Este y el Pacífico, y 65\% más desigual que los países de altos ingresos (véase Grafica 1). Sin embargo, después de aumentar en los años noventa, la desigualdad en Latinoamérica declinó entre 2000 y 2007. De 17 países, de los que se disponía de información comparativa, 12 experimentaron una caída en el coeficiente de Gini. De acuerdo con estos mismos autores, los países en Latinoamérica con mayor desigualdad eran Honduras, Bolivia, Panamá, Brasil, Guatemala, Ecuador, Paraguay, Nicaragua y Chile, que registraron niveles de desigualdad por encima del promedio de la región (52\% de acuerdo con el coeficiente de Gini). Por debajo del promedio en este rubro se encontraban países como México, El Salvador, Perú, Costa Rica, República Dominicana, Argentina, Uruguay y Venezuela (véase Gráfica 2).

Para $\mid$ Zepeda Martínez, Roberto (2013). Neoliberalismo, desempeño económico y mercados 


\section{Grafica 1.- Coeficiente de Gini por región, 2004 (porcentaje)}

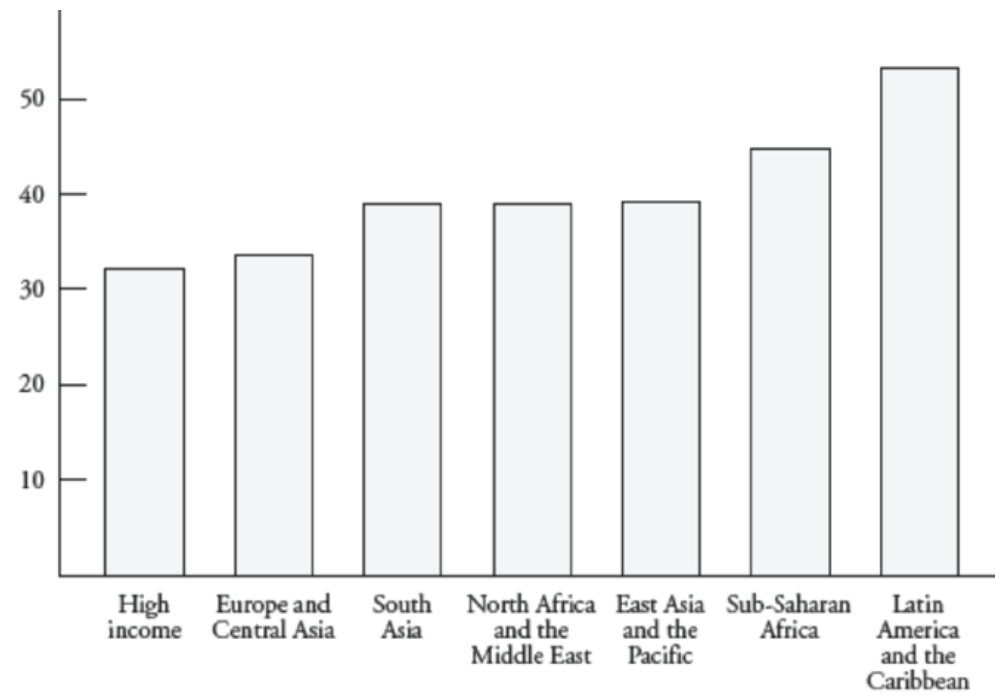

Fuente: López-Calva y Lustig (2010).

\section{Grafica 2.- Latinoamérica: coeficiente de Gini por país, 2007}

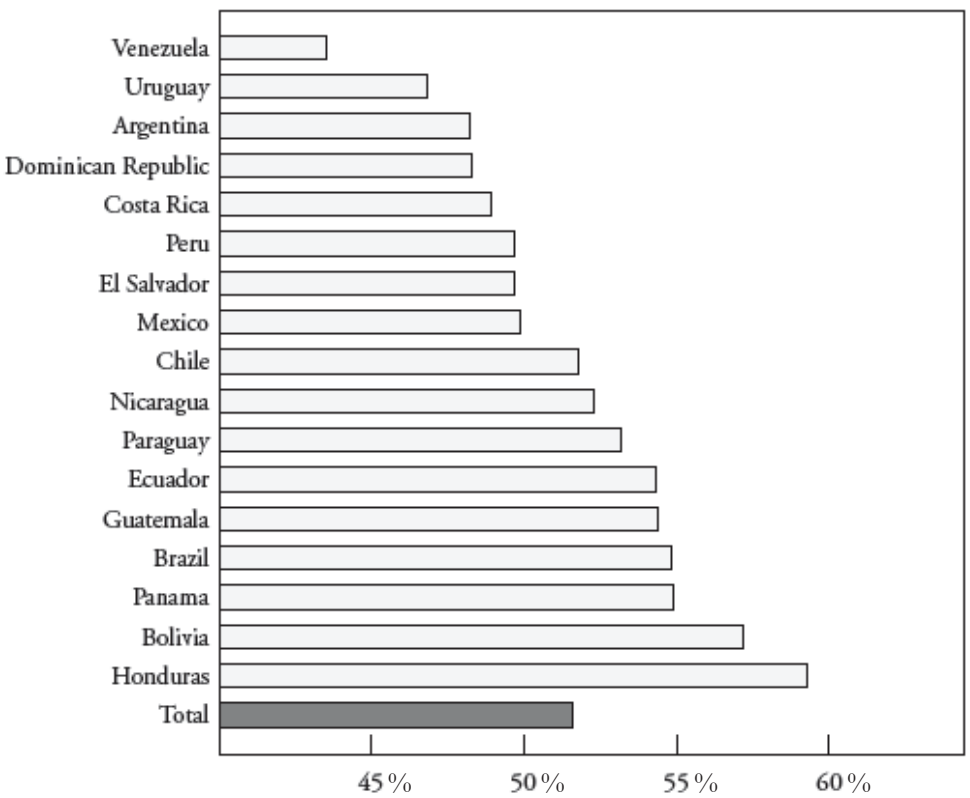

Fuente: Lopez-Calva y Lustig (2010).

Zepeda Martínez, Roberto (2013). Neoliberalismo, desempeño económico y mercados laborales en Latinoamérica: un enfoque comparativo. Ánfora 20(35), 13-40. Universidad Autónoma de Manizales. ISSN 0121-6538. 
La falta de crecimiento económico genera desempleo y reducción de fuentes de trabajo. Por eso, en los períodos de estancamiento económico en Latinoamérica se han acentuado los problemas de los trabajadores al no encontrar un empleo decente. Hacia finales de los 90, la OIT definió el trabajo decente como aquel que brinde oportunidades para que mujeres y hombres disfruten de un empleo productivo y decente, en condiciones de libertad, igualdad, seguridad y dignidad humana. Este concepto tiene cuatro componentes: empleo, protección social, derechos de los trabajadores y diálogo social (ILO, 1999).

La dificultad para obtener empleo decente ha conducido a muchos trabajadores desempleados a la migración y a la economía informal. Esto último se ha presentado en gran medida en México, en donde el número de trabajadores en la economía informal se ha incrementado significativamente durante el periodo 1980-2010. Cifras extraoficiales señalan que hacia 2007, alrededor del 60\% de la PEA estaba localizada en el sector informal. En las siguientes secciones se analizan los niveles de desempleo y de empleo informal en la región.

\section{Desempleo urbano en Latinoamérica}

Con base en información estadística de la OIT (2011, p. 101), hacia 2010, el desempleo urbano en Latinoamérica era más alto en Colombia, Venezuela y Chile, países que registraron tasas de $12.4 \%, 8.7 \%$ y $8.2 \%$, respectivamente. Los niveles más bajos de desempleo urbano en este mismo año fue en Brasil (6.7\%), Bolivia (6.5\%) y México (6.4\%). En cuanto a la evolución del desempleo en este grupo de países, a pesar de que este indicador tuvo niveles más altos en los 90 , disminuyó en la primera década de 2000. Se puede observar que los altos niveles de desempleo urbano se mantuvieron constantes entre 1990 y 2010 en los casos de Colombia (12.6\%), Argentina (11.9\%), Venezuela (11.1\%) y Uruguay (10.3\%). A pesar de que se presentaron fluctuaciones al alza en 1990-2000, el desempleo se redujo entre 2000 y 2010 en Uruguay, Paraguay y Ecuador. Los niveles más bajos de desempleo urbano en el periodo 1990-2010 en este grupo de países, se dieron en México (4.7\%) Brasil (6.5\%) y Bolivia (6.6\%). Mientras que el promedio de los países analizados aquí fue de $8.7 \%$ en los años antes mencionados (Cuadro 3).

Para $\mid$ Zepeda Martínez, Roberto (2013). Neoliberalismo, desempeño económico y mercados 


\section{Cuadro 3.- Desempleo urbano en Latinoamérica. Países selectos: 1990 - 2010}

\begin{tabular}{|l|c|c|c|c|c|c|}
\hline \multicolumn{1}{|c}{ País } & 1990 & 1995 & 2000 & 2005 & 2010 & Promedio \\
\hline Colombia & 10.5 & 8.8 & 17.3 & 13.9 & 12.4 & 12.6 \\
\hline Argentina & 7.5 & 17.5 & 15.1 & 11.6 & 7.7 & 11.9 \\
\hline Venezuela & 10.4 & 10.3 & 13.9 & 12.3 & 8.7 & 11.1 \\
\hline Uruguay & 8.5 & 10.3 & 13.6 & 12.2 & 7.1 & 10.3 \\
\hline Chile & 7.8 & 7.3 & 9.7 & 9.2 & 8.2 & 8.4 \\
\hline Perú & 8.3 & 7.1 & 7.8 & 9.6 & 7.9 & 8.1 \\
\hline Ecuador & 6.1 & 6.9 & 9 & 8.5 & 7.6 & 7.6 \\
\hline Paraguay & 6.6 & 5.3 & 10 & 7.6 & 7.2 & 7.3 \\
\hline Bolivia & 7.3 & 3.6 & 7.5 & 8.2 & 6.5 & 6.6 \\
\hline Brasil & 4.3 & 4.6 & 7.1 & 9.8 & 6.7 & 6.5 \\
\hline México & 2.8 & 6.2 & 3.4 & 4.7 & 6.4 & 4.7 \\
\hline Promedio & 7.3 & 8.0 & 10.4 & 9.8 & 7.9 & 8.7 \\
\hline
\end{tabular}

Fuente: elaboración propia, con datos de la OIT (2011, p. 105).

Los magros resultados en el desempeño económico han impedido la creación suficiente de empleos en el sector formal y han configurado un escenario poco favorable para los trabajadores; asimismo han alentado la búsqueda de nuevos modelos de producción en los sectores industriales. En la siguiente sección se aborda esta paradoja de la economía neoliberal.

Sin embargo, habría que analizar la metodología para medir el desempleo y tomar en cuenta las condiciones laborales de los trabajadores ocupados, ya que gran parte de estos se encuentra laborando en el empleo informal. En este sentido, no se trata de ver si hay o no suficiente creación de empleos o si las tasas de desempleo son muy altas o bajas; lo que realmente es esencial es ver qué tipo de trabajos se están creando y a partir de ahí, llegar a análisis más exactos en el funcionamiento del mercado laboral. Por otra parte, un factor que se debe tomar en cuenta es la metodología para medir el desempleo, ya que así se podría tener 30 un enfoque más real de la situación.

Para $\quad$ Zepeda Martínez, Roberto (2013). Neoliberalismo, desempeño económico y mercados 
La metodología utilizada para medir las tasas de desempleo debe reflejar una situación más exacta de la situación laboral. Por ejemplo, en el caso de México, las encuestas en hogares aplicadas por el Instituto Nacional de Estadística, Geografía e Informática (INEGI) toman en cuenta el término ocupación, en lugar de empleo. De tal manera, cada persona que está ocupada es considerada como empleada. Hay muchas personas que no tienen trabajo asalariado, pero están ocupadas desempeñando alguna actividad, por la cual no reciben remuneración.

En los resultados de las encuestas, que son la base de las estadísticas oficiales, estas personas no están consideradas como desempleadas. Esta situación disminuye significativamente las tasas de desempleo. Por lo tanto, el desempleo debería incluir a todas aquellas personas que no reciben salario por su trabajo, ya sea en el sector formal o informal. Además del desempleo, es necesario identificar el subempleo o empleo informal, ya que este tipo de estadísticas escasean. Un gran porcentaje de la fuerza laboral en México (4 de cada 10) trabaja por su cuenta en lo que pueden, muchos en el comercio ambulante o informal.

Las altas tasas de desempleo afectan las condiciones del empleo existente y lo hacen más precario. El poder de negociación de los trabajadores disminuye cuando el desempleo y subempleo son altos, ya que esto significa que los trabajadores empleados pueden ser más rápidamente reemplazados por lo que Marx llamó "el ejército de reserva" que forman los desempleados. De ahí que en los mercados laborales, los empleadores siempre busquen mantener tasas de desempleo relativamente altas con el propósito de depreciar los salarios; y los sindicatos, por su parte, busquen erradicar el desempleo, ya que a menor desempleo, mayor poder de los sindicatos y mayores salarios para los trabajadores (Campbell et al., 2003, pp. 210-215).

De esta manera, en países latinoamericanos ha disminuido el poder de negociación de los trabajadores y los sindicatos, debido al desempleo estructural creado por el sistema económico donde predomina una economía exportadora, la cual no ha creado el número suficiente de empleos. Además, con la flexibilización del trabajo y de las formas de contratación instrumentadas por los empleadores con el propósito de aumentar la productividad, se ha incentivado la actividad económica creando un cierto tipo de empleos de escasa seguridad para los trabajadores.

Es pertinente destacar que no todos los países latinoamericanos cuentan con el Seguro de Desempleo (una prestación laboral que permite que los desempleados sigan accediendo a una remuneración constante, mientras encuentran un

Para citar este artículo: 
nuevo empleo). Según la OIT (2000, pp. 174-175), 6 de los 11 países incluidos en este análisis, cuentan con algún esquema de Seguro de Desempleo: Argentina, Chile, Uruguay, Brasil, Ecuador y Venezuela. Sin embargo, esta prestación es prácticamente inexistente en México (aunque existe un programa similar en el Distrito federal, aún no ha sido creado un Seguro de Desempleo nacional) y el resto de los países de la región. Tal condición podría explicar la baja tasa de desempleo en México, por ejemplo. Además, en Uruguay, Chile y Argentina, este seguro para los desempleados es aún más completo que en Brasil, Venezuela y Ecuador. De hecho, tres de los países con mayores índices de desempleo urbano en 2007 (Uruguay, Brasil y Argentina) cuentan con seguro de desempleo.

Las cotizaciones de los empleadores y de los trabajadores son la fuente más importante de financiación de seguro de desempleo, con algunas excepciones. En Brasil, se financia con un impuesto sobre los ingresos de las empresas, en Chile con los ingresos fiscales generales y en el Uruguay con las cotizaciones al seguro social y con una fracción al impuesto sobre el valor añadido. El derecho a percibir prestaciones de desempleo es muy limitado. Quedan normalmente excluidos los asalariados más expuestos a rachas de desempleo, como los de la construcción, el servicio doméstico, la agricultura y los trabajadores jóvenes (OIT, 2000, p. 175).

\section{Empleo informal}

El empleo informal en Latinoamérica es una de las características de los mercados laborales y está relacionado con la falta de generación de empleos suficientes debido al bajo crecimiento económico. Como hemos visto en las secciones anteriores, la ausencia de crecimiento económico ha generado altas tasas de desempleo y la reducción del empleo formal. De esta manera, las dificultades enfrentadas por los trabajadores en Latinoamérica se han complicado durante los periodos de estancamiento económico, ya que no pueden encontrar un empleo dentro de la economía formal.

Esta situación, que ha llevado a muchos desempleados a opciones alternativas como el empleo informal, ha acontecido en la mayoría de los países de la región en los que el número de trabajadores en la economía informal se ha incrementado en décadas recientes. El empleo informal en la región se ha disparado en el periodo 1980-2010; aunque no hay estadísticas abundantes y exactas, susceptibles de comparar, se puede señalar que alrededor del 60\% de la fuerza laboral estaba empleada en el sector informal hacia mediados de los 2000s (OIT, 2009, p. 20). 
La proliferación del empleo informal es una de las repercusiones más significativas del neoliberalismo en América Latina. El neoliberalismo ha estimulado un creciente sector informal durante las últimas décadas. En el cuadro 4, se constata que hacia principios de 2000, en Latinoamérica, alrededor del 57\% del empleo no-agrícola se localizaba en el sector informal (Gallin, 2001, p. 533). Sin embargo, el empleo no-agrícola en el sector informal fue más alto en África que en Latinoamérica en este mismo año. Estos niveles de informalidad se pueden corroborar con los datos de la OIT (2009, pp. 19-21) los cuales señalan que el sector informal en 2007 era de 58.6\% en la región latinoamericana. Esta misma fuente destaca que el empleo en el sector informal en el 2007 fue más alto en Perú (73.8\%), Ecuador (71.8\%) y Colombia (61.4\%); mientras que México registró un porcentaje por debajo del promedio (ver cuadro 5).

De esta manera, la informalidad en el empleo se ha expandido en años recientes en la región. Este ha sido uno de los efectos más impactantes del neoliberalismo sobre los mercados laborales en Latinoamérica: la creación de mercados laborales caracterizados por el empleo informal y precario. De igual forma, las reformas neoliberales han acelerado la emergencia de empleos de bajos salarios, principalmente en el sector servicios.

De acuerdo con la OIT (2011, p. 67), para el año 2010, el empleo informal total en la región de Latinoamérica era de 50.4\%, siendo más alto entre las mujeres $(53.7 \%)$ que entre los hombres (47.8\%). Es decir, alrededor de la mitad de la fuerza laboral empleada en Latinoamérica estaba inserta en alguna forma de empleo informal. El $50.4 \%$ de los trabajadores informales se dividían de la siguiente manera: $32.7 \%$ trabajaba en el sector informal de la economía; mientras que $12.3 \%$ laboraba en un empleo informal del sector formal y $5.4 \%$ lo hacía en el sector doméstico.

Como se aprecia en el cuadro 6, hacia el año 2010, los países con mayores niveles de empleo informal en Latinoamérica eran Paraguay (70.4\%), Perú (70.3\%) y Bolivia (69.5\%), que tenían más del 60\% de su fuerza laboral en la informalidad. Después con porcentajes que fluctuaban entre el $50 \%$ y $60 \%$ se encontraban Colombia (59.6\%), Ecuador (56.4\%) y México (54.2\%). Con niveles de informalidad por debajo del promedio de la región (es decir, menos del 50.4\%), estaban Venezuela (47.4\%), Argentina (46.7\%), Brasil (42.1\%) y Uruguay (37.7\%). De las cuatro economías principales de Latinoamérica, dos tenían más de la mitad de su fuerza laboral en la informalidad (Colombia y México), y las otras dos (Brasil y Argentina) tenían menos de la mitad de sus trabajadores en el sector informal.

Para $\mid$ Zepeda Martínez, Roberto (2013). Neoliberalismo, desempeño económico y mercados 


\section{Cuadro 4.- Porcentaje del sector informal en el empleo en \\ Latinoamérica, África y Asia, 2001}

\begin{tabular}{lrcc}
\hline & $\begin{array}{r}\text { Latinoamérica } \\
\text { y el Caribe }\end{array}$ & África & Asia \\
\hline $\begin{array}{l}\text { Empleo total excluyendo } \\
\text { agricultura }\end{array}$ & $15 \%$ & $18 \%$ & $15-30 \%$ \\
$\begin{array}{l}\text { Empleo total incluyendo } \\
\text { agricultura }\end{array}$ & $45 \%$ & $75 \%$ & $75-85 \%$ \\
Empleo no-agrícola & $57 \%$ & $78 \%$ & $45-85 \%$ \\
\hline Empleo urbano & $40 \%$ & $61 \%$ & $40-60 \%$ \\
\hline
\end{tabular}

Fuente: preparado con datos de la OIT, 2009, p. 20.

Cuadro 5.- Sector informal en Latinoamérica, 2007

\begin{tabular}{ll}
\hline América latina & $58.6 \%$ \\
\hline Ecuador & $69.6 \%$ \\
\hline Perú & $68.8 \%$ \\
\hline Colombia & $61.4 \%$ \\
\hline México & $52.7 \%$ \\
\hline Panamá & $40.6 \%$ \\
\hline
\end{tabular}

Fuente: preparado con datos de la OIT, 2009, p. 20.

\section{Cuadro 6.- Empleo informal en Latinoamérica}

\begin{tabular}{|c|c|}
\hline País & 2010 \\
\hline Paraguay & 70.4 \\
\hline Perú & 70.3 \\
\hline Bolivia & 69.5 \\
\hline Colombia & 59.6 \\
\hline Ecuador & 56.4 \\
\hline México & 54.2 \\
\hline Venezuela & 47.4 \\
\hline Argentina & 46.7 \\
\hline Brasil & 42.1 \\
\hline Uruguay & 37.7 \\
\hline Chile & - \\
\hline
\end{tabular}

Fuente: OIT, 2011. 


\section{Sindicalización}

Como resultado del bajo crecimiento económico, las relativas altas tasas de desempleo, y la creciente participación de los trabajadores en el empleo informal, los sindicatos han enfrentado un panorama adverso para aumentar su membresía y los niveles de sindicalización son cada vez menos representativos en los países de Latinoamérica. Como se puede ver en el cuadro 7, la sindicalización en la región presentaba diversos niveles hacia mediados de la primera década de 2000. Sin embargo, Argentina era el país con mayor sindicalización con alrededor del 25\% de densidad sindical, muy por encima de Brasil (17\%), Chile (16\%), y México (10\%). Los sindicatos, los regímenes políticos y la legislación laboral en cada uno de estos países han desempeñado un papel muy relevante para la configuración de este paisaje diverso de la representación sindical.

En cuanto a la evolución de las tasas de sindicalización en el periodo neoliberal, se observa que en la mayoría de los países examinados aconteció una caída en la densidad sindical. En Brasil, la tasa de sindicalización pasó del 32.1\% hacia principios de los 90, al 17\% en la mitad de 2000. Mientras que en Argentina, la reducción de este indicador también fue notable: cayó de $48.7 \%$ a $25.4 \%$ en el periodo 1986-1995. En este nivel de representación sindical se ha mantenido hasta la mitad de 2000. En el caso de México, también se presentó un declive de la densidad sindical, al pasar del 14\% al 10\% entre 1985 y 2005, de acuerdo con datos oficiales. En contraparte, el único país que presentó un aumento en la tasa de sindicalización fue Chile, ya que la tasa de sindicalización pasó del 11.6\% al 16\% entre 1986 y 2005 (OIT, 1998, p. 57; Cardoso, 2004; Aguilar, 2008).

Según los datos recopilados, México era el país con menor sindicalización en la primera mitad de 2000. Sin embargo, hacia la segunda mitad de la década de los 90, Colombia y Perú fueron los países con menor nivel de sindicalización (dentro del grupo examinado aquí), con el 7 y $8 \%$ de representación sindical, respectivamente, de acuerdo con la OIT (2003, p. 65). En términos de sindicalización en Latinoamérica, México está por debajo de Argentina y Brasil, las economías más grandes de la región. La sindicalización en México está incluso por debajo de Chile, país en el que prácticamente se suprimieron los derechos laborales y la libertad para organizar sindicatos durante la dictadura militar de Pinochet. Fue a finales de los 80 cuando los trabajadores se afiliaron en sindicatos de nuevo.

La representación del sector servicios en las economías nacionales en Latinoamérica se incrementó notablemente durante el período 1980-2010. De acuerdo 
con diversos autores, este hecho es una de las causas estructurales del declive sindical, debido a que este sector es más difícil de sindicalizarse y esto impide que la membresía sindical se expanda. Por otra parte, se considera que el bastión fuerte del sindicalismo se encuentra en el sector industrial, el cual en la mayoría de los países de la región ha presentado una tendencia a la baja.

Cuadro 7.- Densidad sindical como porcentaje de la fuerza laboral noagrícola en el periodo neoliberal

\begin{tabular}{|l|c|c|c|}
\hline & $1986-1990$ & $1991-1995$ & $2000-2005(\mathrm{~b})$ \\
\hline Argentina & 48.7 & 25.4 & 25 \\
\hline Bolivia & $\cdot$ & 16.4 & \\
\hline Brasil & $\cdot$ & 32.1 & 17 \\
\hline Chile & 11.6 & 15.9 & 16 \\
\hline Colombia & 11.2 & 7 & \\
\hline Ecuador & $\cdot$ & 10 & 10 \\
\hline México (a) & 14 & 11 & \\
\hline Venezuela & 25.9 & 15 & \\
\hline Uruguay & 19.9 & 12 & \\
\hline Paraguay &. & 9.3 & \\
\hline Perú & & 8 & \\
\hline
\end{tabular}

OIT, 1998, Aguilar, 2008. Datos de Argentina y Brasil 2000-2005, Cardoso (2004). Datos de 2000-2005, Chile, Celhay, 2007.

\section{Conclusiones}

Después del análisis de diversos indicadores en la esfera económica y laboral, derivados de organismos oficiales, se concluye que hay un panorama desfavorable para Latinoamérica en las últimas décadas, en especial en el desempeño de la economía y las características de los mercados laborales. Esta situación persiste a pesar de que la mayoría de los países de la región presenció un viraje hacia un sistema económico neoliberal. Diversas estadísticas muestran un crecimiento económico moderado con crisis recurrentes en ciertos periodos que han impedido la suficiente creación de empleos en la economía formal. Esta situación ha

36 propiciado un sector informal de casi el $60 \%$ en la región. 
No obstante, habría que realizar algunos matices. Respecto a la aplicación de políticas neoliberales, Chile fue el primer país de la región en implementarlas (1976), mientras que en Brasil la reforma neoliberal inició en 1994. Sin embargo, ambos países mostraron un notable crecimiento económico en el periodo 1980-2010. Por lo anterior, no podemos establecer una relación estrecha entre neoliberalismo y desempeño económico. Aunque, al parecer, este dinamismo económico no ha resuelto problemas como la desigualdad social, la cual sigue siendo significativa.

Por otra parte, a pesar de que México ha adoptado el modelo económico neoliberal desde los 80, tuvo un desempeño económico bajo en esa década, aunque en los 90 registró una expansión económica notable. En el 2000, su tasa de crecimiento ha declinado por debajo del total en la región. Argentina ha tenido un comportamiento irregular en la expansión económica y ha sufrido altibajos en dicho periodo. Sin embargo, entre 2003 y 2010, su economía creció en promedio alrededor del $8 \%$, uno de los porcentajes más altos de la región.

Chile es el país que más ha crecido en términos de Producto Interno Bruto durante el periodo 1980-2010, aunque prevalece en este país un nivel de desigualdad por encima del promedio de la región. También en Chile se aplicaron por vez primera las políticas neoliberales en la región, desde la segunda mitad de la década de 1970. Por su parte, Colombia presentó uno de los mayores niveles de crecimiento económico promedio en el periodo 1980-2010 (solo debajo de Chile, en el grupo analizado aquí), aunque también tuvo el más alto nivel de desempleo urbano y presenta un alto grado de empleo informal, mayor que el promedio en la región latinoamericana.

Un análisis más pormenorizado de la evolución del PIB por países permite constatar las drásticas caídas de la economía en México, a mediados de los 90, y en Argentina, a principios de 2000. Mientras que en México se han profundizado las reformas neoliberales después de la crisis económica, en Argentina se ha optado por disminuirlas. En el periodo 1998-2006, el viraje hacia la izquierda en los gobiernos latinoamericanos se dio en una gran parte de Latinoamérica, lo que contribuyó a moderar las políticas neoliberales. Aunque no fue el caso de México.

La proliferación de empleos en el sector informal de la economía también representa una característica de los mercados laborales en Latinoamérica, debido a la escasa creación de empleos en el sector formal. Este ha sido el caso de Ecuador, Perú, Colombia, México y de otros países en la región, que presentan las mayores condiciones de "precarización" del trabajo: empleos en el sector informal, bajos salarios y escasa protección social para un gran porcentaje de 
la fuerza laboral, que se ubica en el 60\%. Este escenario en el mercado laboral plantea nuevos retos para los sindicatos. Uno de los retos del sindicalismo consiste en organizar al enorme grupo, cada vez mayor, de trabajadores informales. La tasa de sindicalización en Latinoamérica es de las más bajas del mundo, muy por debajo de Europa, por ejemplo.

Las políticas neoliberales no han incentivado la expansión de la economía en la región ni tampoco han solucionado el problema del empleo, lo que ha generado mayor inestabilidad para los trabajadores en un mercado laboral caracterizado por altos niveles de informalidad y precariedad, como se ha demostrado aquí por medio de estadísticas oficiales de organismos internacionales y gobiernos nacionales durante las tres últimas décadas.

\section{Referencias}

Aguilar, J. 2008). Globalización, Trabajo y Sindicalismo en México. México: Konrad Adenauer-ITACA-CENPROS.

Cardoso, A. (2004). Industrial relations, social dialogue and employment in Argentina, Brazil and Mexico. Employment Strategy Papers.

Campbell, R. et al (2003). Economía laboral. España: McGraw Hill.

Celhay, P. y Gil, D. (2007). Sindicalización y negociación colectiva. Informe de apoyo para Consejo de Trabajo y Equidad.

CEPAL (2004). Anuario estadístico de América Latina y el Caribe. Santiago de Chile.

CEPAL (2008). Anuario estadístico de América Latina y el Caribe. Santiago de Chile.

CEPAL (2012). Anuario estadístico de América Latina y el Caribe. Santiago de Chile.

Cook, M. L. (2002). Labor reform and dual transitions in Brazil and the Southern cone. Latin American Politics and Society, 44(1), 1-34.

Cook, M. L. (1998). Toward Flexible Industrial Relations? Neoliberalism, Democracy, and Labor Reform in Latin America. Industrial Relations, 37(3), 311-336.

Economic Commission for Latin America and the Caribbean, ECLAC, (2000). Preliminary overview of the economies of Latin America and the Caribbean 2000. Santiago de Chile: United Nations - CEPAL - ECLAC.

Gamble, A. (2001). Neo-liberalism. Capital and Class, (75), 127-34.

Gamble, A. (2000). Politics and fate. Cambridge: Polity Press.

González, F. E. (2008). Dual transitions from authoritarian rule: institutionalized regimes in Chile and Mexico, 1970-2000. Baltimore: Johns Hopkins University Press.

Para citar este artículo:
Zepeda Martínez, Roberto (2013). Neoliberalismo, desempeño económico y mercados laborales en Latinoamérica: un enfoque comparativo. Ánfora 20(35), 13-40. Universidad Autónoma de Manizales. ISSN 0121-6538. 
Hague, R., y Harrop, M. (2007). Comparative government and politics. London: MacMillan. Harvey, D. (2005). A brief history of neoliberalism. Oxford: University Press.

Heywood, A. (2007). Politics. London - New York: Palgrave.

Hirst, P. y G. T. (2003). The future of globalization. En Michie, J. (2003). The handbook of globalization. UK-USA: Edward Elgar Publishing.

International Monetary Fund (IMF) (2012). Recuperado de: http://www.imf.org/external/index.htm octubre de 2012.

Kirby, P. (2003). Introduction to Latin America: twenty-first century challenges. London: Sage.

ILO. (1998). World labor report 1997-1998. Geneva: International labor organization.

MacLeod, D. (2005). Privatization and the limits of state autonomy in Mexico. Rethinking the orthodox paradox. Latin American Perspectives, 32(4), 36-64.

OIT (2000). Informe sobre el trabajo en el mundo 2000. Ginebra, Suiza: OIT.

OIT (2002). Panorama Laboral 2002, América Latina y el Caribe. Perú: Oficina Internacional del Trabajo.

OIT (2007). Panorama Laboral 2007, América Latina y el Caribe. Perú: Oficina Internacional del Trabajo.

OIT (2008). Panorama Laboral 2008, América Latina y el Caribe. Perú: Oficina Internacional del Trabajo.

OIT (2009). Panorama Laboral 2009, América Latina y el Caribe. Perú: Oficina Internacional del Trabajo.

OIT (2010). Panorama Laboral 2010, América Latina y el Caribe. Perú: Oficina Internacional del Trabajo.

OIT (2011). Panorama Laboral 2011, América Latina y el Caribe. Perú: Oficina Internacional del Trabajo.

Robinson, W. (2004). Global crisis and Latin America. Bulletin of Latin American Research, 23(2), 135-153.

Saad-Filho, A. (2005). From Washington Consensus to post-Washington Consensus: Neoliberal agendas for economic development. En A. Saad-Filho. Neoliberalism: a critical reader (pp. 113-119). London: Pluto Press.

Saad-Filho, A. (2005). Neoliberalism: a critical reader. London: Pluto press.

Stallings, B. y Peres, W. (2000). Growth, employment and equity. The impact of the economic reforms in Latin American and Caribbean. Washington: Economic Commission for Latin American and Caribbean, Brookings Institution Press.

Para 
United Nations Conference on Trade and Development (UNCTAD) (2008). Handbook of Statistics, 2008. United Nations.

United Nations Conference on Trade and Development (UNCTAD) (2011). Handbook of Statistics, 2011. United Nations.

Visser, J. (2003). Unions and unionism around the world. En Schnabel y Adison (2003). International Handbook of Trade Unions. Edward Elgar: Cheltenham.

Weyland, K. (2004). Critical debates. Neoliberalism and democracy in Latin America: a mixed record. Latin American Politics \& Society, 46(1), 135-157.

Williamson, O. (2000). What should the World Bank think about the Washington consensus? World Bank Research Observer, 15(2), 251-264.

López-Calva, L. F., y Lustig, N. (2010). Declining Inequality in Latin America: A Decade of Progress? Brookings Inst. Press.

Para $\mid$ Zepeda Martínez, Roberto (2013). Neoliberalismo, desempeño económico y mercados Autónoma de Manizales. ISSN 0121-6538. 